\title{
Association between glucose fluctuation during 2-hour oral glucose tolerance test, inflammation and oxidative stress markers, and $\beta$-cell function in a Chinese population with normal glucose tolerance
}

\author{
Chifa $\mathrm{Ma}^{1} \wedge$, Yiwen $\mathrm{Liu}^{1}$, Shuli $\mathrm{He}^{2}$, Jingbo Zeng ${ }^{3}$, Pingping $\mathrm{Li}^{4,5}$, Chunxiao Ma ${ }^{4,5}$, Fan Ping ${ }^{1}$, \\ Huabing Zhang ${ }^{1}$, Lingling $\mathrm{Xu}^{1}$, Wei $\mathrm{Li}^{1}$, Yuxiu $\mathrm{Li}^{1} \wedge$
}

${ }^{1}$ Department of Endocrinology, Key Laboratory of Endocrinology, Ministry of Health, Peking Union Medical College Hospital, Peking Union Medical College, Chinese Academy of Medical Sciences, Beijing, China; ${ }^{2}$ Department of Nutrition, Peking Union Medical College Hospital, Beijing, China; ${ }^{3}$ Department of Endocrinology, Fuxing Hospital, the Eighth Clinical Medical College, Capital Medical University, Beijing, China; ${ }^{4}$ State Key Laboratory of Bioactive Substance and Function of Natural Medicines, Institute of Materia Medica, Chinese Academy of Medical Sciences and Peking Union Medical College, Beijing, China; ${ }^{5}$ Diabetes Research Center of Chinese Academy of Medical Sciences, Beijing, China

Contributions: (I) Conception and design: C Ma, W Li, Y Li; (II) Administrative support: F Ping, H Zhang, L Xu, W Li, Y Li; (III) Provision of study materials or patients: J Zeng, P Li, C Ma, H Zhang, Y Li; (IV) Collection and assembly of data: C Ma, Y Liu, S He; (V) Data analysis and interpretation: C Ma, Y Liu, S He; (VI) Manuscript writing: All authors; (VII) Final approval of manuscript: All authors.

Correspondence to: Yuxiu Li, MD. Department of Endocrinology, Key Laboratory of Endocrinology, Ministry of Health, Peking Union Medical College Hospital, Peking Union Medical College, Chinese Academy of Medical Sciences, Shuaifuyuan No.1, Dongcheng District, Beijing 100730, China. liyuxiu@medmail.com.cn.

\begin{abstract}
Backgrounds: Glucose fluctuation (GF) may have detrimental effects in individuals with diabetes; however, clinical data on the association between short-term GF, inflammation/oxidative stress markers, and islet $\beta$-cell function based on a population with normal glucose tolerance (NGT) are insufficient. Therefore, we aimed to explore these associations in a Chinese population of 209 individuals with NGT in a crosssectional analysis.
\end{abstract}

Methods: Individuals were categorized based on GF tertiles, calculated as the maximum-minimum glucose levels among four time points (0, 30, 60, $120 \mathrm{~min}$ ) during 2-hour oral glucose tolerance test (OGTT). Plasma inflammation markers tumor necrosis factor- $\alpha$ (TNF- $\alpha$ ) and interleukin-6 (IL-6), and oxidative stress markers superoxide dismutase (SOD), and 8-oxo-2'-deoxyguanosine (8-oxo-dG) were measured. Islet $\beta$-cell function was estimated according to the disposition index (DI) at the early (30 min) and total (120 min) phase of the OGTT, adjusted for insulin sensitivity.

Results: Individuals in the middle and highest tertile of GF had reduced $\beta$-cell function, and increased plasma SOD and TNF- $\alpha$ levels compared with those in the lowest tertile of $\mathrm{GF}(\mathrm{P}<0.05)$. Multiple linear regression analysis indicated that GF was positively associated with TNF- $\alpha, 8$-oxo-dG and SOD levels, but negatively associated with $\beta$-cell function, whereas IL-6, TNF- $\alpha, 8$-oxo-dG and SOD levels were negatively associated with $\beta$-cell function $(\mathrm{P}<0.05)$.

Conclusions: GF may increase inflammation and oxidative stress markers in individuals with NGT, which could contribute to reduced $\beta$-cell function. Thus, maintaining glucose stability after a meal may have beneficial effects on delaying $\beta$-cell dysfunction, suggesting that diet and exercise strategies to decrease diet related GF are warranted.

Keywords: $\beta$-cell function; glucose fluctuation; inflammation; oxidative stress

^ ORCID: Chifa Ma, 0000-0003-4148-2758; Yuxiu Li, 0000-0001-7500-0855. 
Submitted Aug 27, 2020. Accepted for publication Nov 26, 2020.

doi: $10.21037 /$ atm-20-6119

View this article at: http://dx.doi.org/10.21037/atm-20-6119

\section{Introduction}

Type 2 diabetes mellitus (T2DM), characterized by progressive deterioration of islet $\beta$-cell function, imposes a heavy social economic and public health burden globally. Individuals with impaired glucose tolerance (IGT) and impaired fasting glucose (IFG) are considered to be at greater risk for developing future T2DM than those with normal glucose tolerance (NGT); however, a large proportion of individuals with NGT could also develop diabetes after 5 years or longer (1). Moreover, mounting evidence indicates a degree of $\beta$-cell dysfunction in individuals with prediabetes (including IFG and IGT), as well as in some individuals with NGT (2-4). Therefore, it is an urgent task to identify the diabetes risk factors of individuals with NGT to implement effective and timely interventions that can prevent or delay the development of diabetes.

Diabetes and prediabetes may be diagnosed based on the value of fasting plasma glucose (FPG) and/or 2-hour postprandial plasma glucose (PPG) after an oral glucose tolerance test (OGTT). A high plasma glucose level can cause $\beta$-cell dysfunction by changing the pulsatility pattern of insulin secretion (5). Moreover, high glucose levels lead to upregulated expression of ATP-conducting mitochondrial outer membrane voltage-dependent anion channel-1 (VDAC1), resulting in cellular ATP depletion and impaired glucose-stimulated insulin secretion (6). Although overexpressed VDAC1 does not cause islet apoptosis itself, high VDAC1 levels combined with a high glucose concentration $(20 \mathrm{Mm})$, markedly increase the apoptosis rate (6). In recent years, there has been increasing awareness of the clinical value in assessment of glucose fluctuation (GF), which is regarded as a type of glycemic disorder, separate from high FPG and PPG, present in individuals with diabetes and prediabetes (7). GF is closely associated with oxidative stress and an inflammatory response, which may in turn aggravate $\beta$-cell dysfunction and diabetic complications (8-12). A German study based on a population with T2DM suggested that glycemic variability was strongly related to postprandial $\beta$-cell function for individuals taking oral hypoglycemia drugs (13). Another study using an in vitro model further demonstrated that intermittent high glucose could promote $\beta$-cell dysfunction by increasing oxidative stress, leading to apoptosis (14). GF was also suggested to have harmful effects to endothelial function in both healthy controls and in individuals with T2DM (15). In most clinical studies, GF is estimated based on the mean amplitude of glycemic excursions (MAGE) computed from 3-day continuous glucose monitoring, however, the most common short-term GF may occur after a meal. Indeed, Polonsky and colleagues calculated the short-term GF by subtracting the minimum from the maximum glucose level during the 2-hour OGTT, which was associated with the plasma levels of oxidative stress markers in both individuals with diabetes and NGT (16). Another study used the difference between the maximum and minimum glucose levels during the 4-hour OGTT as a GF indicator in patients with coronary artery disease, which was closely associated with $\beta$-cell function and inflammation markers (8). Thus, the difference between maximum and minimum glucose levels during the 2-hour OGTT may be an accurate short-term indicator of GF and could have important downstream effects for the body.

Although most previous studies on GF have focused on its role in diabetes, GF is also closely associated with future incident cardiovascular disease and malignancies in the nondiabetic population $(17,18)$, demonstrating the need for more attention to be paid to the role of GF in individuals with NGT. Moreover, identifying the risk factors of diabetes in individuals with NGT will help in devising strategies for delaying the development of diabetes. Toward this end, the aim of the present study was to explore the association between short-term GF, estimated as the maximumminimum glucose levels during the 2-hour OGTT, plasma inflammation and oxidative stress markers, and $\beta$-cell function in a Chinese population with NGT. These findings can provide more evidence on the role of short-term GF in the development and progression of diabetes.

We present the following article in accordance with the MDAR checklist and the STROBE reporting checklist (available at http://dx.doi.org/10.21037/atm-20-6119).

\section{Methods}

\section{Study population and clinical measurements}

This study was based on a T2DM project conducted in the 


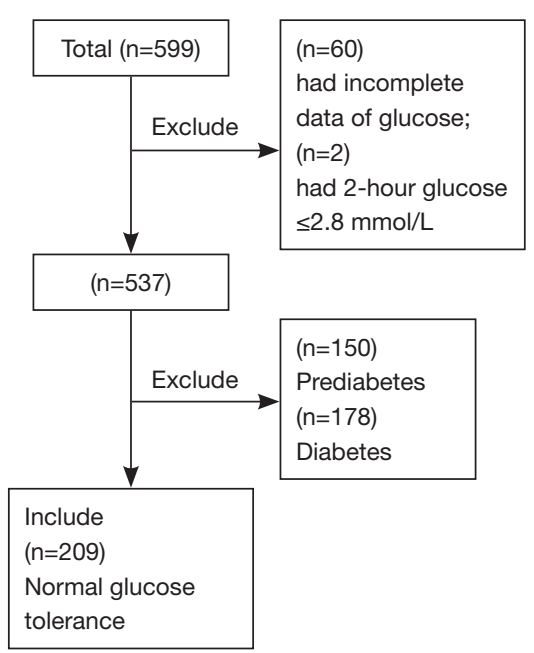

Figure 1 The flow chart of the population-based study.

Changping district of Beijing from 2014 to 2015, which is described in detail elsewhere (19). A total of 599 participants were recruited and completed questionnaires related to baseline characteristics, including age, gender and medical history. Detailed physical examination including body mass index (BMI) and blood pressure, were also performed. After a 2-hour 75-g OGTT, diabetes and prediabetes were diagnosed in accordance with the 1999 World Health Organization criteria. Participants diagnosed with diabetes or prediabetes, a 2 -hour $\mathrm{PPG} \leq 2.8 \mathrm{mmol} / \mathrm{L}$, or missing data of glucose levels were excluded from the present analysis, whereas some individuals with missing data of markers of inflammation [tumor necrosis factor- $\alpha(\mathrm{TNF}-\alpha, \mathrm{n}=35)$ and interleukin-6 (IL-6, n=36)] and oxidative stress [8-oxo-2'deoxyguanosine (8-oxo-dG, $\mathrm{n}=36$ ) and superoxide dismutase (SOD, $\mathrm{n}=35)]$ were nevertheless included in the analysis. Finally, a total of 209 adults of Chinese Han ethnicity aged from 19 to 73 years with NGT (FPG $<6.1 \mathrm{mmol} / \mathrm{L}$ and 2-hour PPG $<7.78 \mathrm{mmol} / \mathrm{L}$ ) were enrolled in the study (Figure 1), including 64 men and 145 women.

The study was conducted in accordance with the Declaration of Helsinki (as revised in 2013). The study was approved by the Ethics Committee of Peking Union Medical College Hospital (Approval Number: ZS-1274) and informed consent was taken from all individual participants.

\section{Biochemical measurements}

Blood samples for plasma glucose measurement were obtained at $0,30,60$, and 120 minutes after a 75 -g oral glucose load. Plasma glucose concentrations were assayed using a glucose oxidase assay. Insulin concentrations were quantified by a Chemiluminescent enzyme immunoassay (ADVIA Centaur XP, Siemens) according to the manufacturer instructions. Hemoglobin A1c (HbA1c) was analyzed in whole blood using high-performance liquid chromatography (D10 Hemoglobin Testing System, BioRad). An automated analyzer was used to determine serum uric acid (UA) and lipids concentrations. Plasma inflammation markers (TNF- $\alpha$ and IL-6) and oxidative stress markers (8-oxo-dG and SOD) were determined by respective kits (Cloud-Clone Corp, Houston, TX, USA).

\section{Variables and calculation}

Basal insulin resistance was estimated by homeostatic model assessment of insulin resistance (HOMA-IR):

HOMA-IR $=\frac{\text { Fasting INS }(\mathrm{uIU} / \mathrm{mL}) \times \text { FPG }(\mathrm{mmol} / \mathrm{L})}{22.5}(20)$.

Basal and glucose stimulated insulin sensitivity was estimated by Matsuda insulin sensitivity index: $\left(\operatorname{ISI}_{M}\right)$ :

$$
\text { ISI }_{\mathrm{M}}=\frac{10000}{\sqrt{\begin{array}{l}
(\text { Mean OGTT glucose concentration }[\mathrm{mg} / \mathrm{dL}] \times \\
\text { Mean OGTT insulin concentration }(\mathrm{uIU} / \mathrm{mL}))
\end{array}}}
$$

The disposition index (DI) was used as the measure of $\beta$-cell function adjusted by insulin sensitivity, calculated in the early phase at $30 \mathrm{~min}\left(\mathrm{DI}_{30}\right)$ : $\mathrm{DI}_{30}=\left(\frac{\text { InsulinAUC30 }}{\text { GlucoseAUC30 }}\right) \times \mathrm{ISI}_{\mathrm{M}}$, and in the total phase at $120 \min \left(\mathrm{DI}_{120}\right): \mathrm{DI}_{120}=\left(\frac{\text { InsulinAUC120 }}{\text { GlucoseAUC120 }}\right) \times \operatorname{ISI}_{\mathrm{M}}(22)$.

\section{Statistical analysis}

Normally distributed quantitative variables are presented as the mean \pm standard deviation (SD), and categorical variables are presented as number (percentage). Nonnormally distributed variables are presented as the median [interquartile range (IQR)] or transformed using the natural logarithm as appropriate. Quantitative parameters were compared among three groups using the Kruskal-Wallis test or one-way analysis of variance with a post-hoc Bonferroni test. Differences among three groups were evaluated by the $\chi^{2}$ test for categorical variables. Correlations between GF, inflammation and oxidative stress markers, and $\beta$-cell 
secretion and insulin resistance index were analyzed using Spearman's correlation coefficient. Multiple linear regression analysis was used to explore the associations between GF, inflammation and oxidative stress markers, and $\beta$-cell function. Model 1 was adjusted for age, gender, BMI and systolic blood pressure (SBP). Model 2 was adjusted for the covariates of model 1 plus triglycerides (TG), highdensity lipoprotein cholesterol (HDL-C) and UA. Model 3 was adjusted for the covariates of model 2 plus the 2-hour PPG. $\beta$ coefficients and $95 \%$ confidence intervals (CIs) were calculated per 1-SD increment of GF, and inflammation and oxidative stress markers. Individuals with missing data of markers of inflammation and oxidative stress were not included in analyses involving the related markers.

Statistical analyses were conducted using SPSS 22.0 software. A two-sided $\mathrm{P}$ value $<0.05$ was considered statistically significant.

\section{Results}

\section{Association of GF with clinical and demographic characteristics}

Clinical and demographic characteristics of the participants based on tertiles of GF are shown in Table 1. The median GF of individuals was $2.37 \mathrm{mmol} / \mathrm{L}$ (IQR, 1.54-2.80), $4.07 \mathrm{mmol} / \mathrm{L}$ (IQR, 3.76-4.49) and $5.96 \mathrm{mmol} / \mathrm{L}$ (IQR, 5.34-7.38) in the lowest, middle and highest tertile, respectively. Compared with individuals in the lowest GF tertile, those in the highest tertile were more likely to be older, male, and also had higher levels of UA and HbA1c (all $\mathrm{P}<0.05)$. In terms of $\beta$-cell function, individuals in higher GF tertile had lower $\mathrm{DI}_{30}$ and $\mathrm{DI}_{120}$ values $(\mathrm{P}<0.001)$ than those in the lowest tertile of GF, whereas there were no significant differences in insulin resistance index among the three GF groups.

Individuals with higher GF also exhibited higher values of plasma TNF- $\alpha$ and SOD than those in the lowest GF tertile $(\mathrm{P}<0.05)$, whereas the differences in IL-6 and 8 -oxo-dG among the three groups did not reach statistical significance.

\section{Correlations of GF, inflammation and oxidative stress markers, and $\beta$-cell function and insulin resistance index}

The correlations between GF, inflammation and oxidative stress markers, and $\beta$-cell function and insulin resistance index are provided in Table 2. GF was negatively correlated with $\mathrm{DI}_{30}(\mathrm{r}=-0.493, \mathrm{P}<0.001)$ and $\mathrm{DI}_{120}(\mathrm{r}=-0.431, \mathrm{P}<0.001)$ The level of plasma IL-6 was also negatively correlated with both $\mathrm{DI}_{30}(\mathrm{r}=-0.152, \mathrm{P}=0.046)$ and $\mathrm{DI}_{120}(\mathrm{r}=-0.210$, $\mathrm{P}=0.005)$, whereas the plasma TNF- $\alpha$ level was only negatively correlated with $\mathrm{DI}_{120}(\mathrm{r}=-0.183, \mathrm{P}=0.016)$. In terms of oxidative stress markers, 8 -oxo-dG was negatively correlated with $\mathrm{DI}_{30}(\mathrm{r}=-0.152, \mathrm{P}=0.046)$ and $\mathrm{SOD}$ was negatively correlated with $\mathrm{DI}_{120}(\mathrm{r}=-0.187, \mathrm{P}=0.014)$. Neither the inflammation markers nor the oxidative stress markers were correlated with the insulin resistance index.

\section{Multiple linear regression analysis of the association between glucose fluctuation and $\beta$-cell function}

The coefficients of multiple linear regression analysis for GF and $\beta$-cell function estimated by $\mathrm{DI}_{30}$ and $\mathrm{DI}_{120}$ are presented in Table 3. GF was negatively associated with $\mathrm{DI}_{30}$ $(\beta=-29.818,95 \%$ CI: -39.519 to $-20.118, \mathrm{P}<0.001)$ and $\mathrm{DI}_{120}(\beta=-20.676,95 \%$ CI: -28.870 to $-12.482, \mathrm{P}<0.001)$ in Model 1 after adjustment for age, gender, BMI and SBP. With further adjustment for TG, HDL-C and UA in Model 2, the negative trend remained significant $(\mathrm{P}<0.001)$. After additional adjustment for 2-hour PPG in Model 3, the negative association between GF and $\beta$-cell function remained significant $(\mathrm{P}<0.001)$.

\section{Multiple linear regression analysis of the association between $G F$ and markers of inflammation and oxidative stress}

The coefficients of multiple linear regression analysis for GF and inflammation markers are provided in Table 4. After adjustment for age, gender, BMI and SBP, GF was positively associated with TNF- $\alpha$ in Model 1 ( $\beta=2.204,95 \%$ CI: 0.719 to $3.689, \mathrm{P}=0.004)$, and the association remained significant ( $\beta=2.314,95 \%$ CI: 0.786 to $3.842, \mathrm{P}=0.003$ ) independent of age, gender, BMI, SBP, lipids, UA and 2-hour PPG in Model 3. IL-6 was also positively associated with GF, but the $\mathrm{P}$ value did not reach statistical significance in any model (all $\mathrm{P}>0.05$, Mode 1 to 3 ).

The coefficients of multiple linear regression analysis for GF and oxidative stress markers are provided in Table 5 . After adjustment for age, gender, BMI and SBP, GF was positively associated with SOD ( $\beta=4.178,95 \%$ CI: 1.341 to 7.105, $\mathrm{P}=0.004)$ and 8 -oxo-dG ( $\beta=6.240,95 \% \mathrm{CI}: 0.835$ to 11.646, $\mathrm{P}=0.024)$ in Model 1. These associations remained significant independent of age, gender, BMI, SBP, lipids, UA and 2-hour PPG in Model $3(\mathrm{P}<0.05)$. 
Table 1 Association of glucose fluctuation with Clinical and demographic characteristics

\begin{tabular}{|c|c|c|c|c|}
\hline & Lowest GF $(n=69)$ & Middle GF $(n=71)$ & Highest GF (n=69) & $\mathrm{P}$ \\
\hline Gender, n (\%) & & & & $<0.001$ \\
\hline Female & $56(81.2)$ & $56(78.9)$ & $33(47.8)$ & \\
\hline Male & $13(18.8)$ & $15(21.1)$ & $36(52.2)$ & \\
\hline BMI $\left(\mathrm{kg} / \mathrm{m}^{2}\right)$ & $24.87 \pm 3.46$ & $25.72 \pm 3.65$ & $25.44 \pm 3.6$ & 0.356 \\
\hline $\mathrm{SBP}(\mathrm{mmHg})$ & $121.06 \pm 16.98$ & $126.96 \pm 17.96$ & $126.96 \pm 17.24$ & 0.073 \\
\hline $\mathrm{DBP}(\mathrm{mmHg})$ & $76.49 \pm 9.64$ & $75.82 \pm 10.32$ & $74.87 \pm 9.31$ & 0.619 \\
\hline TC (mmol/L) & $5.13 \pm 0.95$ & $5.40 \pm 1.19$ & $5.39 \pm 0.91$ & 0.215 \\
\hline LDL-C (mmol/L) & $2.58 \pm 0.72$ & $2.78 \pm 0.78$ & $2.79 \pm 0.66$ & 0.179 \\
\hline UA (umol/L) & $262.64 \pm 74.09$ & $282.33 \pm 79.62$ & $298.82 \pm 75.75^{\star}$ & 0.023 \\
\hline HbA1c (\%) & $5.2(5.0-5.5)$ & $5.4(5.2-5.6)$ & $5.5(5.15-5.7)^{\star}$ & 0.009 \\
\hline FPG (mmol/L) & $5.42(5.13-5.65)$ & $5.57(5.37-5.76)$ & $5.43(5.26-5.80)$ & 0.081 \\
\hline 2h-PPG (mmol/L) & $6.11(5.11-6.83)$ & 6.28 (5.69-7.15) & $5.90(4.62-6.83)$ & 0.054 \\
\hline LnHOMA-IR & $0.77 \pm 0.43$ & $0.84 \pm 0.47$ & $0.71 \pm 0.67$ & 0.353 \\
\hline $\operatorname{Ln}\left(\mathrm{ISI}_{\mathrm{M}}\right)$ & $1.59 \pm 0.42$ & $1.43 \pm 0.46$ & $1.42 \pm 0.64$ & 0.105 \\
\hline $\mathrm{Dl}_{30}$ & $229.89 \pm 82.97$ & $174.12 \pm 45.55^{\star}$ & $151.77 \pm 70.92^{*}$ & $<0.001$ \\
\hline $\mathrm{SOD}(\mathrm{U} / \mathrm{mL})(\mathrm{n}=174)$ & $56.41(45.63-66.08)$ & $60.16(53.47-70.13)$ & $62.52(55.34-75.12)^{\star}$ & 0.030 \\
\hline
\end{tabular}

Data were presented as mean $\pm \mathrm{SD}, \mathrm{n}(\%)$ or median (interquartile range). ${ }^{*}$ Compared with the lowest tertile of GF, a two-side $\mathrm{P}<0.05$ was considered statistically significant; " Compared with the middle tertile of GF, a two-side $\mathrm{P}<0.05$ was considered statistically significant. GF, glucose fluctuation; BMI, body mass index; SBP, systolic blood pressure; DBP, diastolic blood pressure; TC, total cholesterol; TG, triglycerides; HDL-C, high-density lipoprotein cholesterol; LDL-C, low-density lipoprotein cholesterol; UA, uric acid; HbA1c, hemoglobin A1c; FPG, fasting plasma glucose; PPG, postprandial plasma glucose; HOMA-IR, homeostatic model assessment of insulin resistance; ISIM, Matsuda insulin sensitivity index; TNF- $\alpha$, tumor necrosis factor- $\alpha$; IL-6, interleukin-6; 8-oxo-dG, 8-oxo-2'-deoxyguanosine; SOD, superoxide dismutase.

\section{Multiple linear regression analysis of the association between inflammation/ oxidative stress markers and $\beta$-cell function}

The coefficients of multiple linear regression analysis for inflammation/oxidative stress markers and $\beta$-cell function are provided in Table 6.TNF- $\alpha$ was negatively associated with $\mathrm{DI}_{120}(\beta=-9.455,95 \% \mathrm{CI}:-17.895$ to $-1.015, \mathrm{P}=0.028)$, whereas IL-6 was negatively associated with both $\mathrm{DI}_{30}(\beta$ $=-10.933,95 \%$ CI: -21.812 to $-0.053, \mathrm{P}=0.049)$ and $\mathrm{DI}_{120}$ ( $\beta=-11.672,95 \% \mathrm{CI}:-20.025$ to $-3.319, \mathrm{P}=0.006)$ after adjustment for age, gender, BMI and SBP in Model 1. These associations remained valid after adjustment for TG, HDL-C and UA in Model 2 and with further adjustment for 2-hour PPG in Model 3. 
Table 2 Correlations of glucose fluctuation, inflammation and oxidative stress markers, and $\beta$-cell function and insulin sensitivity index

\begin{tabular}{|c|c|c|c|c|c|c|c|c|}
\hline & \multicolumn{2}{|c|}{$\mathrm{Dl}_{30}$} & \multicolumn{2}{|c|}{$\mathrm{Dl}_{120}$} & \multicolumn{2}{|c|}{ LnHOMA-IR } & \multicolumn{2}{|c|}{ LnISIM } \\
\hline GF $(n=209)$ & -0.493 & $<0.001$ & -0.431 & $<0.001$ & -0.032 & 0.643 & -0.134 & 0.052 \\
\hline TNF- $\alpha(n=174)$ & -0.136 & 0.073 & -0.183 & 0.016 & -0.086 & 0.260 & 0.008 & 0.912 \\
\hline IL-6 $(n=173)$ & -0.152 & 0.046 & -0.210 & 0.005 & 0.025 & 0.746 & 0.027 & 0.725 \\
\hline $\operatorname{SOD}(n=174)$ & -0.109 & 0.153 & -0.187 & 0.014 & -0.097 & 0.205 & 0.024 & 0.756 \\
\hline
\end{tabular}

A two-side $\mathrm{P}<0.05$ was considered statistically significant. HOMA-IR, homeostatic model assessment of insulin resistance; ISIM, Matsuda insulin sensitivity index; GF, glucose fluctuation; TNF- $\alpha$, tumor necrosis factor- $\alpha$; IL-6, interleukin-6; 8-oxo-dG, 8-oxo-2'-deoxyguanosine; SOD, superoxide dismutase.

Table $3 \beta$ coefficients and $95 \%$ confidence intervals of multiple linear regression models of glucose fluctuation with $\beta$-cell function

\begin{tabular}{|c|c|c|c|c|}
\hline & \multicolumn{2}{|l|}{$\mathrm{Dl}_{30}$} & \multicolumn{2}{|l|}{$\mathrm{Dl}_{120}$} \\
\hline Model 1 & $-29.818(-39.519,-20.118)$ & $<0.001$ & $-20.676(-28.870,-12.482)$ & $<0.001$ \\
\hline Model 2 & $-30.006(-39.653,-20.359)$ & $<0.001$ & $-20.347(-28.550,-12.144)$ & $<0.001$ \\
\hline Model 3 & $-35.964(-44.814,-27.115)$ & $<0.001$ & $-26.205(-33.418,-18.991)$ & $<0.001$ \\
\hline
\end{tabular}

Model 1 was adjusted for age, gender, body mass index and systolic blood pressure. Model 2 was adjusted for the covariates of model 1 plus triglycerides, high-density lipoprotein cholesterol and uric acid. Model 3 was adjusted for the covariates of model 2 plus 2 -hour postprandial plasma glucose. A two-side $\mathrm{P}<0.05$ was considered statistically significant. $\beta$ coefficients and $95 \%$ confidence intervals $(\mathrm{Cl})$ were calculated as per 1-SD increment of glucose fluctuation.

Table $4 \beta$ coefficients and $95 \%$ confidence intervals of multiple linear regression models of glucose fluctuation with inflammation markers

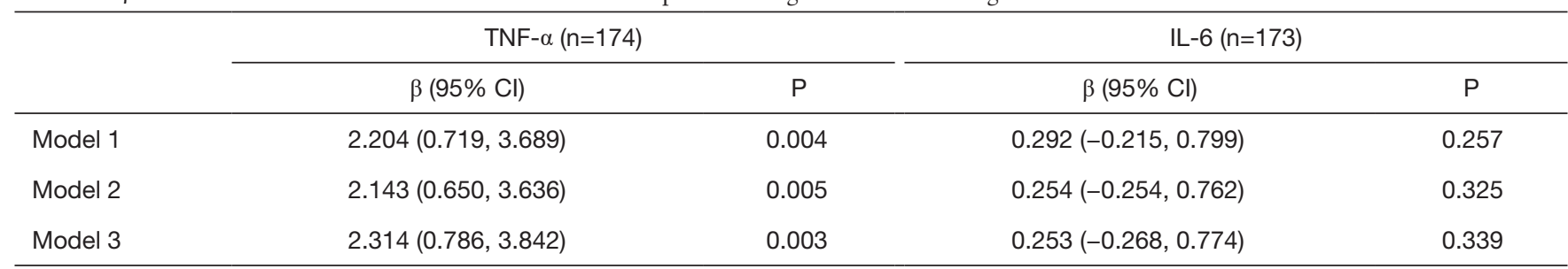

TNF- $\alpha$, tumor necrosis factor- $\alpha$; IL-6, interleukin-6. Model1 was adjusted for age, gender, body mass index and systolic blood pressure. Model 2 was adjusted for the covariates of model 1 plus triglycerides, high-density lipoprotein cholesterol and uric acid. Model 3 was adjusted for the covariates of model 2 plus 2-hour postprandial plasma glucose. A two-side $\mathrm{P}<0.05$ was considered statistically significant. $\beta$ coefficients and $95 \%$ confidence intervals $(\mathrm{Cl})$ were calculated as per 1 -SD increment of glucose fluctuation.

SOD was negatively associated with $\mathrm{DI}_{120}$ in all three models. Moreover, 8-oxo-dG was marginally (but not significantly) associated with $\mathrm{DI}_{30}$ after adjustment for age, gender, BMI and SBP in Model 1 (Table 6). With further adjustment for TG, HDL-C and UA in Model 2, the associations between 8 -oxo-dG and $\mathrm{DI}_{30}$ still did not reach statistical significance $(\mathrm{P}=0.109)$. However, the negative association between $\mathrm{DI}_{30}$ and 8-oxo-dG became significant after additional adjustment for 2-hour PPG $(\beta=-11.269$, 95\% CI: -21.917 to $-0.620, \mathrm{P}=0.038$ ) in Model 3) (Table 6).

\section{Discussion}

Our present study based on individuals with NGT 
Table $5 \beta$ coefficients and $95 \%$ confidence intervals of multiple linear regression models of glucose fluctuation with oxidative stress markers

\begin{tabular}{|c|c|c|c|c|}
\hline & \multicolumn{2}{|c|}{ 8-oxo-dG (n=173) } & \multicolumn{2}{|c|}{$\operatorname{SOD}(n=174)$} \\
\hline Model 1 & $6.240(0.835,11.646)$ & 0.024 & $4.178(1.341,7.105)$ & 0.004 \\
\hline Model 2 & $6.057(0.652,11.461)$ & 0.028 & $4.082(1.237,6.927)$ & 0.005 \\
\hline Model 3 & $5.654(0.140,11.167)$ & 0.045 & $4.564(1.662,7.467)$ & 0.002 \\
\hline
\end{tabular}

8-oxo-dG, 8-oxo-2'-deoxyguanosine; SOD, superoxide dismutase Model 1 was adjusted for age, gender, body mass index and systolic blood pressure. Model 2 was adjusted for the covariates of model 1 plus triglycerides, high-density lipoprotein cholesterol and uric acid. Model 3 was adjusted for the covariates of model 2 plus 2-hour postprandial plasma glucose. A two-side $\mathrm{P}<0.05$ was considered statistically significant. $\beta$ coefficients and $95 \%$ confidence intervals $(\mathrm{Cl})$ were calculated as per $1-\mathrm{SD}$ increment of glucose fluctuation.

Table $6 \beta$ coefficients and $95 \%$ confidence intervals of multiple linear regression models of inflammation and oxidative stress markers with $\beta$-cell function

\begin{tabular}{|c|c|c|c|c|}
\hline & \multicolumn{2}{|l|}{$\mathrm{Dl}_{30}$} & \multicolumn{2}{|l|}{$\mathrm{Dl}_{120}$} \\
\hline \multicolumn{5}{|c|}{ TNF- $\alpha(n=174)$} \\
\hline Model 1 & $-5.574(-16.573,5.424)$ & 0.318 & $-9.455(-17.895,-1.015)$ & 0.028 \\
\hline Model 2 & $-6.520(-17.597,4.558)$ & 0.247 & $-9.245(-17.683,-0.807)$ & 0.032 \\
\hline \multicolumn{5}{|c|}{ IL-6 (n=173) } \\
\hline Model 1 & $-10.933(-21.812,-0.053)$ & 0.049 & $-11.672(-20.025,-3.319)$ & 0.006 \\
\hline Model 2 & $-11.333(-22.339,-0.327)$ & 0.044 & $-10.669(-19.080,-2.258)$ & 0.013 \\
\hline Model 3 & $-11.732(-22.172,-1.292)$ & 0.028 & $-11.038(-18.796,-3.279)$ & 0.006 \\
\hline Model 2 & $-9.137(-20.322,2.048)$ & 0.109 & $-5.127(-14.079,3.826)$ & 0.260 \\
\hline Model 3 & $-11.269(-21.917,-0.620)$ & 0.038 & $-7.355(-15.512,0.803)$ & 0.077 \\
\hline \multicolumn{5}{|c|}{$\operatorname{SOD}(n=174)$} \\
\hline Model 1 & $-9.276(-20.175,1.623)$ & 0.095 & $-9.891(-18.164,-1.618)$ & 0.019 \\
\hline Model 2 & $-8.529(-19.569,2.510)$ & 0.129 & $-9.240(-17.543,-0.936)$ & 0.029 \\
\hline Model 3 & $-7.079(-17.651,3.492)$ & 0.188 & $-7.897(-15.636,-0.158)$ & 0.046 \\
\hline
\end{tabular}

TNF- $\alpha$, tumor necrosis factor- $\alpha$; IL-6, interleukin-6; 8-oxo-2'-deoxyguanosine; SOD, superoxide dismutase Model 1 was adjusted for age, gender, body mass index and systolic blood pressure. Model 2 was adjusted for the covariates of model 1 plus triglycerides, high-density lipoprotein cholesterol and uric acid. Model 3 was adjusted for the covariates of model 2 plus 2-hour postprandial plasma glucose. A two-side $\mathrm{P}<0.05$ was considered statistically significant. $\beta$ coefficients and $95 \%$ confidence intervals $(\mathrm{Cl})$ were calculated as per $1-\mathrm{SD}$ increment of inflammation and oxidative stress markers.

demonstrated that individuals in the higher tertiles of short-term GF (using the difference between maximum and minimum glucose at various time points during the 2 -hour OGTT) had reduced $\beta$-cell function but higher plasma levels of TNF- $\alpha$ and SOD compared with those of individuals in the lowest GF tertile. Moreover, multiple linear regression analysis indicated that GF was negatively associated with $\beta$-cell function but positively associated with 
TNF- $\alpha$, SOD and 8-oxo-dG levels, and these inflammation and oxidative stress markers were also negatively associated with $\beta$-cell function. Although the positive association between IL- 6 and GF did not reach statistical significance, IL-6 was also negatively associated with $\beta$-cell function. In addition, individuals in the lowest GF tertile were more likely to be female and younger (mean age 46.42 years), which may be related to high estrogen levels that have been shown to contribute to a decrease in oxidative stress and inflammation (23-25). However, multiple linear regression analysis indicated that gender and age were not significantly associated with inflammation or oxidative stress markers in the whole population (data not shown).

Both experimental and clinical studies have established that GF was closely associated with $\beta$-cell function (13,14,26-28); however, most of these clinical studies included only individuals with diabetes and GF was estimated according to MAGE, which is typically calculated from 3-day continuous glucose monitoring. Nevertheless, Murata et al. (8) found that GF during the 4-hour OGTT was closely associated with $\beta$-cell function in patients with different degrees of glucose tolerance and coronary artery disease, suggesting that short-term GF may be another potential marker to estimate $\beta$-cell function. Our present study including only individuals with NGT provides further evidence that estimation of shorter-term GF during the 2 -hour OGTT was significantly associated with $\beta$-cell function, which may be the main mechanism related to oxidative stress and inflammation. Increased oxidative stress was observed in the Goto-Kakizaki rat diabetes model, displaying high basal glucose levels and diminished glucosestimulated insulin release both in vivo and in vitro $(29,30)$. Individuals with abnormal glucose tolerance also show altered levels of oxidative stress markers such as SOD and 8-oxo-dG. SOD is a type of antioxidant, and its levels were shown to be increased in individuals with T2DM compared with those of healthy controls, which is considered to indicate a compensatory mechanism in response to elevated oxidative stress (31). 8-oxo-dG is a product of DNA oxidative damage, which was also shown to be elevated in subjects with abnormal glucose tolerance (19). Moreover, a clinical study indicated that GF caused a significant increase in oxidative stress and endothelial dysfunction compared with a constant high glucose state in both healthy controls and T2DM patients (15). Our present data support these previous studies by showing a positive association of GF during the 2-hour OGTT with SOD and 8-oxo-dG in individuals with NGT, and these oxidative stress markers were negatively associated with $\beta$-cell function. Therefore, short-term GF may be also one cause of $\beta$-cell dysfunction in individuals with NGT via oxidative stress. This also supports the previous in vitro results that intermittent high glucose had more of an impact on $\beta$-cell dysfunction than sustained hyperglycemia due to increased oxidative stress (14). We also found that the levels of TNF- $\alpha$, as an inflammatory cytokine, were positively associated with GF but negatively associated with $\beta$-cell function in individuals with NGT, which is consistent with a study in diabetic rats showing that GF enhanced the expression of inflammation factors such as TNF- $\alpha$ (32) as another critical signal linked to $\beta$-cell dysfunction (33). Since the present cross-sectional analysis can only demonstrate that the negative association between GF and $\beta$-cell function was closely linked to inflammation and oxidative stress, larger longitudinal mechanistic studies are needed to confirm the causal relationships. Nevertheless, these findings suggested that diet and exercise strategies to maintain glucose stability after a meal would be warranted to prevent $\beta$-cell dysfunction.

In addition to the aggravation of $\beta$-cell dysfunction, GF has been shown to play critical roles in the occurrence and development of diabetic complications $(12,34)$. Glucose variability was an independent risk for future incident cardiovascular disease even in individuals without diabetes (18). Interestingly, we found that GF was independently associated with oxidative stress and inflammation markers in patients with NGT, which may have detrimental effects promoting the development of metabolic disorders. From this point of view, diet and exercise intervention to regulate GF, and ultimately decrease inflammation and oxidative stress to delay $\beta$-cell dysfunction, should be a general recommendation, irrespective of an individual's glucose tolerance status.

These findings therefore offer a new perspective for diabetes prevention and treatment, while drawing attention to the role of GF after a meal. Our study also had some limitations. First, the cross-sectional design makes it difficult to yield a causal relationship, and longitudinal mechanistic studies are warranted. Nevertheless, the major results are supported by previous clinical and preclinical studies $(14,15,29-33)$. Second, we only included Chinese Han individuals, and thus further study is needed to explore whether the results apply to other ethnicities. However, restricting the sample to a single race also reduced the 
possibility of introducing other confounding factors common in multiethnic studies.

\section{Conclusions}

In conclusion, as a simple indicator of GF, the difference between maximum and minimum glucose levels during the 2-hour OGTT was positively associated with inflammation and oxidative stress markers but negatively associated with $\beta$-cell function in a Chinese population with NGT. GF may increase plasma inflammation and oxidative stress markers in individuals with NGT, which could contribute to reduced $\beta$-cell function. Thus, maintaining glucose stability after a meal may have beneficial effects even in individuals with NGT, and diet and exercise strategies to decrease diet related GF are warranted to delay or prevent the development of diabetes.

\section{Acknowledgments}

Funding: This study was funded by CAMS Innovation Fund for Medical Sciences (CIFMS) (CIFMS2016-I2M-4-001) and the Non-profit Central Research Institute Fund of Chinese Academy of Medical Sciences (No. 2017PT32020, No. 2018PT32001, No. 2019PT320007).

\section{Footnote}

Reporting Checklist: The authors have completed the MDAR checklist and the STROBE reporting checklist. Available at http://dx.doi.org/10.21037/atm-20-6119

Data Sharing Statement: Available at http://dx.doi. org/10.21037/atm-20-6119

Conflicts of Interest: All authors have completed the ICMJE uniform disclosure form (available at http://dx.doi. org/10.21037/atm-20-6119). The authors declare that they have no conflicts of interest.

Ethical Statement: The authors are accountable for all aspects of the work in ensuring that questions related to the accuracy or integrity of any part of the work are appropriately investigated and resolved. The study was conducted in accordance with the Declaration of Helsinki (as revised in 2013). The study was approved by the Ethics Committee of Peking Union Medical College Hospital
(Approval Number: ZS-1274) and informed consent was taken from all individual participants.

Open Access Statement: This is an Open Access article distributed in accordance with the Creative Commons Attribution-NonCommercial-NoDerivs 4.0 International License (CC BY-NC-ND 4.0), which permits the noncommercial replication and distribution of the article with the strict proviso that no changes or edits are made and the original work is properly cited (including links to both the formal publication through the relevant DOI and the license). See: https://creativecommons.org/licenses/by-nc-nd/4.0/.

\section{References}

1. Unwin N, Shaw J, Zimmet P, et al. Impaired glucose tolerance and impaired fasting glycaemia: the current status on definition and intervention. Diabet Med 2002;19:708-23.

2. Gastaldelli A, Ferrannini E, Miyazaki Y, et al. Betacell dysfunction and glucose intolerance: results from the San Antonio metabolism (SAM) study. Diabetologia 2004;47:31-9.

3. Ferrannini E, Gastaldelli A, Miyazaki Y, et al. beta-Cell function in subjects spanning the range from normal glucose tolerance to overt diabetes: a new analysis. J Clin Endocrinol Metab 2005;90:493-500.

4. Yang Y, Wang M, Tong J, et al. Impaired GlucoseStimulated Proinsulin Secretion Is an Early Marker of beta-Cell Impairment Before Prediabetes Stage. J Clin Endocrinol Metab 2019;104:4341-6.

5. Salehi A, Qader SS, Grapengiesser E, et al. Inhibition of purinoceptors amplifies glucose-stimulated insulin release with removal of its pulsatility. Diabetes 2005;54:2126-31.

6. Zhang E, Mohammed Al-Amily I, Mohammed S, et al. Preserving Insulin Secretion in Diabetes by Inhibiting VDAC1 Overexpression and Surface Translocation in beta Cells. Cell Metab 2019;29:64-77.e6.

7. Monnier L, Colette C, Owens DR. Glycemic variability: the third component of the dysglycemia in diabetes. Is it important? How to measure it? J Diabetes Sci Technol 2008;2:1094-100.

8. Murata M, Adachi H, Oshima S, et al. Glucose fluctuation and the resultant endothelial injury are correlated with pancreatic beta cell dysfunction in patients with coronary artery disease. Diabetes Res Clin Pract 2017;131:107-15.

9. Wu N, Shen H, Liu H, et al. Acute blood glucose 
fluctuation enhances rat aorta endothelial cell apoptosis, oxidative stress and pro-inflammatory cytokine expression in vivo. Cardiovasc Diabetol 2016;15:109.

10. Monnier L, Colette C. Glycemic variability: should we and can we prevent it? Diabetes Care 2008;31 Suppl 2:S150-4.

11. Kohnert KD, Freyse EJ, Salzsieder E. Glycaemic variability and pancreatic beta-cell dysfunction. Curr Diabetes Rev 2012;8:345-54.

12. Zhang ZY, Miao LF, Qian LL, et al. Molecular Mechanisms of Glucose Fluctuations on Diabetic Complications. Front Endocrinol (Lausanne) 2019;10:640.

13. Kohnert KD, Augstein P, Zander E, et al. Glycemic variability correlates strongly with postprandial beta-cell dysfunction in a segment of type 2 diabetic patients using oral hypoglycemic agents. Diabetes Care 2009;32:1058-62.

14. Shao C, Gu J, Meng X, et al. Systematic investigation into the role of intermittent high glucose in pancreatic betacells. Int J Clin Exp Med 2015;8:5462-9.

15. Ceriello A, Esposito K, Piconi L, et al. Oscillating glucose is more deleterious to endothelial function and oxidative stress than mean glucose in normal and type 2 diabetic patients. Diabetes 2008;57:1349-54.

16. Polonsky KS, Licinio-Paixao J, Given BD, et al. Use of biosynthetic human C-peptide in the measurement of insulin secretion rates in normal volunteers and type I diabetic patients. J Clin Invest 1986;77:98-105.

17. Kobayashi D, Noto H, Takahashi O, et al. Glycemic variability and subsequent malignancies among the population without diabetes. Diabetes Res Clin Pract 2020;159:107987.

18. Yu JH, Han K, Park S, et al. Effects of long-term glycemic variability on incident cardiovascular disease and mortality in subjects without diabetes: A nationwide populationbased study. Medicine (Baltimore) 2019;98:e16317.

19. Zhou MC, Zhu L, Cui X, et al. Reduced peripheral blood mtDNA content is associated with impaired glucosestimulated islet beta cell function in a Chinese population with different degrees of glucose tolerance. Diabetes Metab Res Rev 2016;32:768-74.

20. Matthews DR, Hosker JP, Rudenski AS, et al. Homeostasis model assessment: insulin resistance and beta-cell function from fasting plasma glucose and insulin concentrations in man. Diabetologia 1985;28:412-9.

21. Matsuda M, DeFronzo RA. Insulin sensitivity indices obtained from oral glucose tolerance testing: comparison with the euglycemic insulin clamp. Diabetes Care 1999;22:1462-70.
22. Stancakova A, Javorsky M, Kuulasmaa T, et al. Changes in insulin sensitivity and insulin release in relation to glycemia and glucose tolerance in 6,414 Finnish men. Diabetes 2009;58:1212-21.

23. Delgobo M, Agnes JP, Goncalves RM, et al. $\mathrm{N}$-acetylcysteine and alpha-lipoic acid improve antioxidant defenses and decrease oxidative stress, inflammation and serum lipid levels in ovariectomized rats via estrogen-independent mechanisms. J Nutr Biochem 2019;67:190-200.

24. Kumar R, Balhuizen A, Amisten S, et al. Insulinotropic and antidiabetic effects of 17beta-estradiol and the GPR30 agonist G-1 on human pancreatic islets. Endocrinology 2011;152:2568-79.

25. Balhuizen A, Kumar R, Amisten S, et al. Activation of G protein-coupled receptor 30 modulates hormone secretion and counteracts cytokine-induced apoptosis in pancreatic islets of female mice. Mol Cell Endocrinol 2010;320:16-24.

26. Bian H, Gao X, Gao J. Relationship between glucose fluctuation and beta cell function in patients with diabetes. Zhonghua Yi Xue Za Zhi 2009;89:664-8.

27. Takai M, Anno T, Kawasaki F, et al. Association of the Glycemic Fluctuation as well as Glycemic Control with the Pancreatic beta-cell Function in Japanese Subjects with Type 2 Diabetes Mellitus. Intern Med 2019;58:167-73.

28. Kim M, Chung H, Yoon C, et al. Increase of INS-1 cell apoptosis under glucose fluctuation and the involvement of FOXO-SIRT pathway. Diabetes Res Clin Pract 2012;98:132-9.

29. Mosen H, Salehi A, Alm P, et al. Defective glucosestimulated insulin release in the diabetic Goto-Kakizaki (GK) rat coincides with reduced activity of the islet carbon monoxide signaling pathway. Endocrinology 2005;146:1553-8.

30. Salehi A, Meidute Abaraviciene S, Jimenez-Feltstrom J, et al. Excessive islet NO generation in type 2 diabetic GK rats coincides with abnormal hormone secretion and is counteracted by GLP-1. PLoS One 2008;3:e2165.

31. Tavares AM, Silva JH, Bensusan CO, et al. Altered superoxide dismutase- 1 activity and intercellular adhesion molecule 1 (ICAM-1) levels in patients with type 2 diabetes mellitus. PLoS One 2019;14:e216256.

32. Wang H, Deng J, Chen L, et al. Acute glucose fluctuation induces inflammation and neurons apoptosis in hippocampal tissues of diabetic rats. J Cell Biochem 2019. doi: 10.1002/jcb.29523.

33. Pei P, Yao X, Jiang L, et al. Inorganic arsenic induces 
pyroptosis and pancreatic beta cells dysfunction through stimulating the IRE1alpha/TNF-alpha pathway and protective effect of taurine. Food Chem Toxicol 2019; 125:392-402.

Cite this article as: Ma C, Liu Y, He S, Zeng J, Li P, Ma C, Ping F, Zhang H, Xu L, Li W, Li Y. Association between glucose fluctuation during 2-hour oral glucose tolerance test, inflammation and oxidative stress markers, and $\beta$-cell function in a Chinese population with normal glucose tolerance. Ann Transl Med 2021;9(4):327. doi: 10.21037/atm-20-6119
34. Nusca A, Tuccinardi D, Albano M, et al. Glycemic variability in the development of cardiovascular complications in diabetes. Diabetes Metab Res Rev 2018;34:e3047. 